\title{
Meta
}

Journal des traducteurs

Translators' Journal

Kocourek, Rostilav, Cormier, Monique C. et Lise Lapierre (réd.) (1994) : « Langue et terminologie spécialisées. Specialized

Language and Terminology ", Actes du Colloque

terminologique des étudiants de $2^{\mathrm{e}}$ et $3^{\mathrm{e}}$ cycles, $X \mathrm{XV}^{\mathrm{e}}$ Congrès

international des linguistes, Québec (Québec), Canada, 1992-08-13, Initiales/Initials, vol.14, Halifax (Nouvelle-Écosse), Université Dalhousie, 123 p.

\section{Jean Quirion}

Volume 40, numéro 4, décembre 1995

URI : https://id.erudit.org/iderudit/003900ar

DOI : https://doi.org/10.7202/003900ar

Aller au sommaire du numéro

Éditeur(s)

Les Presses de l'Université de Montréal

ISSN

0026-0452 (imprimé)

1492-1421 (numérique)

Découvrir la revue

Citer ce compte rendu

Quirion, J. (1995). Compte rendu de [Kocourek, Rostilav, Cormier, Monique C. et Lise Lapierre (réd.) (1994) : « Langue et terminologie spécialisées. Specialized Language and Terminology ", Actes du Colloque terminologique des étudiants de $2^{\mathrm{e}}$ et $3^{\mathrm{e}}$ cycles, $\mathrm{XV}^{\mathrm{e}}$ Congrès international des linguistes, Québec (Québec), Canada, 1992-08-13, Initiales/Initials, vol.14, Halifax (Nouvelle-Écosse),

Université Dalhousie, 123 p.] Meta, 40(4), 670-673.

https://doi.org/10.7202/003900ar d'utilisation que vous pouvez consulter en ligne. 
KOCOUREK, Rostislav, CORMIER, Monique C. et Lise LAPIERRE (réd.) (1994) : "Langue et terminologie spécialisées. Specialized Language and Terminology», Actes du Colloque terminologique des étudiants de $2^{c}$ et $3^{c}$ cycles, $\mathrm{XV}^{e}$ Congrès international des linguistes, Québec (Québec), Canada. 1992-08-13, Initiales/Initials, vol. 14, Halifax (Nouvelle-Écosse), Université Dalhousie, $123 \mathrm{p}$.

Le $X V^{c}$ Congrès international des linguistes s'est tenu a l'Université Laval en août 1992. L'événement a servi de cadre à la présentation d'un colloque terminologique d'étudiants de $2^{\mathrm{c}}$ et $3^{\mathrm{c}}$ cycles. Huit communications y ont été prononcées.

Le texte des conférences a été publié dans Initiales/Initials, revue du Département d'études françaises de l'Université Dalhousie, à Halifax. Coordonné par Rostislav Kocourek, professeur à l'Université Dalhousie. Lise Lapierre, étudiante de doctorat à cette même université, et Monique C. Cormier, professeur à l'Université de Montréal, le volume $14 \mathrm{~d}$ 'Initiales/Initials reprend le thème du colloque : "Langue et terminologie spécialisées".

Les actes du colloque étudiant s'ouvrent sur la valeur liée à l'utilisation d'un temps dans un texte scientifique, communication prononcée par Lise Lapierre. La proportion des verbes au présent dans le texte technoscientifique contemporain varie entre $70 \%$ et $90 \%$, estime R. Kocourek. C'est à partir de cette observation que l'auteur s'est intéressé au statut diachronique du présent dans les textes techniques et scientifiques. Afin d'observer l'évolution de l'utilisation des temps dans l'histoire, l'auteur a étudié le présent dans un texte du siècle demier, les Leşons sur les phénomènes de la vie communs aux animaux e't aux légétaux de Claude Bernard. Tentant de cerner la valeur intrinsèque du présent. L. Lapierre fait appel à la grammaire traditionnelle et à la perspective de l'énonciation d'Émile Benveniste. Si la première offre des pistes plutôt vagues, la seconde se révèle plus satisfaisante. L'auteur discute de la valeur de vérité associée au présent et conclut que la «fonction principale du présent consiste à signaler le caractère commentatif de ce qui est dit, c'est-à-dire l'adhérence du locuteur à son énoncé".

Dans sa communication intitulée «Pour le griffage des mots non marqués», René Tondji-Simen aborde la question des marques d'usage géographiques dans les dictionnaires français. Par rapport à quelle norme marque-t-on? R. Tondji-Simen réfute l'idée d'une langue centrale, francilienne. Il rappelle que le français n'est parlé par personne : 
une langue est en effet constituée de la totalité de ses dialectes ; tous les francophones parlent donc un des dialectes du français. Fort de cette constatation, R. Tondji-Simen se demande alors sur quelle base le lexicographe décide de la marque d'un mot. Puis, il propose une grille de marquage. Il suggère de distinguer langue commune et langues particulières. Ainsi, les mots du fonds français commun seraient non griffés. Ce sont les mots appartenant exclusivement à l'une ou l'autre des régions francophones qui seraient marqués. La France serait ainsi traitée sur le même pied que les autres pays de la francophonie. Enfin, pour préciser le griffage, l'auteur propose un marquage à deux degrés. Une première marque identifierait le territoire national (France, Côte-d'Ivoire. Sénégal, etc.). Une seconde marque viendrait au besoin préciser l'aire d'utilisation (Bretagne, Bouaké. Sérères. etc.).

Emmanuel Aitokhuehi étudie dans sa communication la tension entre l'emprunt et la néologie de souche française. Rappelant l'importance du lexique dans la langue, il estime que l'emprunt doit être étudié sous l'éclairage de la lexicologie. Il cite Trescases, qui affirme que le lexique constitue la partie la plus visible, la plus malléable d'une langue et de par la susceptible aux influences profondes. Ecartant de son propos l'emprunt intralinguistique, E. Aitokhuehi discute des définitions de l'emprunt tirées de quelques auteurs et revoit les critères d'identification de l'emprunt. L'étudiant, bien documenté, souligne que l'emprunt pénètre tant la terminologie que la langue générale et distingue les caractéristiques de ces dernières. Il étudie ensuite le fonctionnement et les rapports du terme d'emprunt face au néologisme. Après avoir revu les définitions du néologisme, l'auteur propose un tour d'horizon complet des moyens et méthodes de la formation lexicale et de la formation terminologique. Enfin, l'effet déstabilisateur de la néologie calquée ou empruntée fait l'objet d'une évaluation. Pondéré, l'auteur conclut à la nécessité du juste milieu, rejetant «l'emprunt excessif [et] le purisme stérile en ce qui concerne les débats relatifs à l'emprunt et au néologisme".

La terminologie française des domaines de pointe s'inspire de l'anglais. Celle opinion, généralisée, se base sur le constat suivant : l'anglais est le véhicule de diffusion. sinon de création. des nouvelles technologies. Or, dans le cadre de travaux en néologic, Juan Carlos Rivas remet en question la justesse de ce raisonnement attribuant a l'anglais la paternité par alliance des termes français. Il prend comme objet d'étude l'emprunt et le calque dans le domaine des virus informatiques. Son hypothèse de travail se résume comme suit : la terminologie française de ce sous-domaine de l'informatique ne dérive pas de l'anglais ; les deux terminologies se sont plutôt développées parallèlement autour d'un terme genèse, celui de virus. L'auteur de l'article passe malheureusement sous silence les motifs qui lui permettent de croire que virus jouit du statut de terme noyau. Après examen d'un corpus. J. C. Rivas conclut que les signifiants français résultent d'un emprunt intralinguistique entre les domaines de la médecine et de l'informatique. À cause de l'interdisciplinarité grandissante des développements scientifiques et techniques. l'étudiant avance que l'emprunt intralinguistique est en voie d'accélération.

Nina Hopkins Butlin s'intéresse aux rapports entre discours littéraire et scientifique. Elle s'est attachée à comparer deux textes descriptifs. Le Traité de zoologie, dirigé par P.-P. Grassé et paru en 1951. a été retenu comme texte scientifique : le second, littéraire, s'intitule La paix chez les bêtes, écrit par Colette et publié en 1916. À des fins de comparaison. l'auteur retient des passages où l'on traite du même insecte : le papillon. L'étude des textes confirme les différences attendues entre les deux types de textes. La terminologie, l'objectivité et le public visé - des spécialistes - marquent le texte technique. Quant au texte littéraire, il est caractérisé par la subjectivité, une faible densité terminologique, un style riche. Rien de bien nouveau jusqu'ici. Cependant, N. H. Butlin note une remarquable ressemblance quant à la volonté descriptive. Les deux textes 
partagent des faits empiriques, un vocabulaire riche et précis. Selon Butlin. la science tend à remplacer l'omniscience, et les auteurs de textes scientifiques relativisent de plus en plus leurs connaissances. Leurs textes porteront ainsi davantage leur marque personnelle. C'est ce qui amène l'auteur à souligner que le texte scientifique, à l'instar du texte littéraire, porte un sens largement négociable. Le scientifique émaille son texte de marques subjectives devant la beauté de certains papillons. Ses fréquentes références historiques s'écartent plutôt du descriptif. Quant au texte de Colette. distinctement métaphorique, fortement scientifique, il se révèle également réflectif et narratif. Dans ce texte. c'est derrière le narratif que le lecteur découvre le discours descriptif.

«Dans quel sens peut-on dire qu'un terme technique est monosémique ?» titre Aloysius (Obiukwu. Le jeu de mots cache un propos sérieux : le mode de signification des termes scientifiques et techniques. L'auteur cherche à savoir si l'opposition connotation/ dénotation. proposée par Galisson et Coste. s’applique à la langue de spécialité autant qu’à la langue générale. La dénotation renvoie à la signification primaire du mot. La connotation est un sens que le mot ne porte pas intrinsèquement, mais qui lui est attribué de façon évocatrice. A. Obiukwu examine successivement les arguments en faveur de la monosémie et de la polysémie de la terminologie scientifique et technique. La spécialisation par domaines et l'origine savante des confixés, courante dans les langues de spécialité, vont dans le sens de la monosémie. L'emprunt de mots étrangers favorise également l'univocité. Or, un même terme, lorsqu'utilisé dans plus d'un domaine, acquiert une naturelle polysémie. Mais son utilisation dans un contexte donné désambiguiise le terme scientifique et technique. L'auteur conclut que la distinction monosémie/ polysémie s'opère à partir du niveau de spécialisation du terme et de sa fréquence d'emploi. Mais ces critères sont flous, variables. A. Obiukwu avance que le contexte fait foi de tout et que l'intelligence, l'intérêt personnel et l'observation importent largement dans cette détermination.

Dans l'article suivant, Jean Fontaine présente une synthèse des efforts entrepris pour uniformiser le codage informatique des données terminologiques el lexicographiques. L'étudiant décrit d'abord le langage SGML, acronyme de «Standard Generalized Markup Language". Le SGML (norme ISO 8879) est un puissant outil de structuration des données textuelles. Le langage permet d'uniformiser la codification de la structure d'un document, ce qui facilite grandement les échanges de données entre systèmes. L'auteur décrit de façon brève et complète la logique sous-tendant le SGML. Les marqueurs utilisés dans le SGML sont ensuite décrits: déclarations de marquage, marqueurs descriptifs, références aux entités, déclarations et instructions de traitement. Divers formats d'échanges conformes à la norme du SGML intéressent ensuite J. Fontaine. Leurs possibilités et limites sont étudiées. clairement et simplement, exemples à l'appui. Tout d'abord, MATER, norme sur le format d'échange de données entre gros ordinateurs, est brièvement décrite. Cette norme a une jumelle en micro-informatique. MicroMATER. également exposée. L'étudiant présente ensuite le Nordic Terminological Record Format. utilisé dans les pays scandinaves. Enfin, TEI-TERM (Text Encoding Initiative-TERM) est le dernier format d'échange de données terminologiques examiné. Notons que lors du dernier congrès mondial de la FIT, a Brighton (Grande-Bretagne). le Terminology Interchange Format (TIF) a été retenu comme format d'échange privilégié par les langagiers du monde entier.

Le dernier texte, plutôt original, prend la forme d'un dialogue imaginaire. H. Peter Edwards sattache au rapport qu'entretiennent les textes du critique littéraire Mikhail Bakhtine avec ceux d'académiciens et de scientifiques. Très fantaisiste, la communication présente un dialogue entre Bakhtine et un chercheur sur l'épistémologie. 
Les colloques étudiants, du type de celui présenté au XVe Congrès international des linguistes, doivent se multiplier. Ils favorisent la formation et l'encadrement de la relève. comme le souligne Monique $\mathrm{C}$. Cormier dans son texte de présentation. En prime, la publication des actes entraîne une saine émulation et assure une diffusion élargie des recherches en cours. Il s'agit pour plusieurs participants d'une première présentation devant leurs collègues ; leurs présentations et leurs travaux, volontairement soumis à la critique. en sortiront améliorés. Il faut se féliciter du dynamisme des étudiants et de la multitude des recherches en cours.

JEAN QUIRION

Moneróal. Canada 